\title{
Multifaceted bioinspired hyperbranched polyurethane nanocomposite as a non-contact triggered self-healing material
}

\author{
R. Duarah, A. Deka, N. Karak ${ }^{*}$ \\ Advanced Polymer and Nanomaterial Laboratory, Department of Chemical Sciences, Tezpur University, 784028 Napaam, \\ Assam, India
}

Received 20 June 2019; accepted in revised form 22 September 2019

\begin{abstract}
The current investigation reports in situ fabrication of hyperbranched polyurethane (HPU) nanocomposites with different weight percentages of functionalized silica nanoparticle as nano-reinforcing material. Silica nanoparticles were functionalized with sodium dodecyl sulfate and polyethylene glycol using a facile, simple one-pot method. The nanomaterial and the HPU nanocomposites were assessed by Fourier Transform Infrared Spectroscopy (FTIR), Ultraviolet (UV)-visible, X-ray Diffraction (XRD), Transmission Electron Microscope (TEM), mechanical and thermal studies. The fabricated nanocomposites demonstrated notable tensile strength (25.8 MPa), excellent elongation at break (1495\%), outstanding toughness $\left(340.88 \mathrm{MJ} \cdot \mathrm{m}^{-3}\right)$, good scratch hardness $(7.5 \mathrm{~kg})$, significant impact strength $\left(19.02 \mathrm{~kJ} \cdot \mathrm{m}^{-1}\right)$ and sufficient improvement in hydrophobicity $\left(105.2^{\circ}\right.$ from $\left.76.7^{\circ}\right)$. They also demonstrated remarkable non-contact triggered thermo-responsive shaperecovery (97.6-99.4\%). Moreover they displayed efficient self-healing behavior within just $20 \mathrm{~s}$ under microwave (360 W). The nanocomposites also exhibited biodegradation by bacterial strains, Pseudomonas aeruginosa, and Bacillus subtilis. Thus, the present work promotes this biodegradable nanocomposite as a potential high performing self-healing and selfcleaning material.
\end{abstract}

Keywords: nanocomposites, hyperbranched polyurethane, smart polymers, functionalized silica nanoparticles, biodegradable materials

\section{Introduction}

In recent years, self-cleaning polymeric surfaces have gained substantial impetus for numerous prospective applications including anti-fouling, anti-corrosion, self-cleaning, anti-icing and drag reduction due to their ability to protect against water-borne and dust contaminants $[1,2]$. However, their material performance in an open environment is frequently debatable, consequently, restraining their various practical applications. Moreover, surfaces that can maintain low surface energy (hydrophobic characteristics) during in situ applications to attain durable self-cleaning property is still a challenge. Again, low surface energy gets distorted by external aspects like sun bleaching, strong acid attack, and mechanical damage $[3,4]$. Therefore, the fabrication and design of nano-structured self-healing (maintaining the low surface energy irrespective of external factors) polymeric materials with increased sustainability, excellent energy efficiency, and well-defined robustness have attracted considerable interest within the scientific society in recent times [5]. The healing of polymeric materials is based on the fact that the damaged exposed surface can retain its native hydrophobicity by adjusting its surface energy. Hence, self-healing polymers have attracted definite curiosity in recent 
years as they offer huge prospects where long-standing mechanical durability in defectively available parts is vital [6]. Numerous healing techniques have existed for autonomic restoration of nanoscale/microscopic cracks and defects, but materials that undergo large-scale damage have not been comprehended. Thus, mechanically-durable physical re-mending methods and hydrophobic materials have been developed, but autonomous recovery of hydrophobicity is far more sought-after [7, 8]. Puretskiy et al. [9] fabricated a self-healing hydrophobic surface by combination of fluorinated crystalline fusible wax and colloidal particles. Again, Puretskiy et al. [10] demonstrated self-healing porous coatings that can self-heal through the migration of low surface energy fluoroalkylsilane to the outermost layer. Thus, the importance of restoring structural and mechanical attributes of multiple healing areas of self-healing materials is still in its formative years regardless of their importance as a competent protective layer. In this context, we aim to develop a bioinspired hydrophobic material, with high weathering resistance that can effortlessly regenerate and undergo self-healing like naturally occurring surfaces in nature. Previous literature cites that introduction of hydrophobic binding agents and modifiers of different types of materials such as tetraethyl orthosilicate (TEOS), polytetrafluoroethylene (PTFE) particles, zinc oxide ( $\mathrm{ZnO}$ ), candle soot, fumed silica and titania nano-particles, oligomeric silsesquioxane (POSS) nanoparticles, graphene oxide, graphene, etc. are often used for the fabrication of superhydrophobic self-healing coatings [11-13]. Although such materials exhibit excellent superhydrophobicity and robustness, the majority of the reported superhydrophobic surfaces with multifunctional properties are not fabricated cost-effectively. Moreover, the natural weathering process and accumulation of contaminants gradually decreases the water-repellent capacity of the artificial superhydrophobic surface [14]. Thus, it remains a big task to develop a novel cost-effective and selfhealing hydrophobic polymeric surface with durable mechanical properties, easy to apply over numerous substrates and the ability to sustain under complex external environmental conditions.

In this context, silica nanoparticle(s) (SNP) are one of the most widely used nanoparticles for the production of various nanocomposites. The main advantages of using SNP are comparatively easy production methods, large surface area, smooth nanoporous surface, high thermostability, and good compatibility with polymer matrix [15]. Apart from these, incorporation of SNP into a polymer matrix improves most of the mechanical and physical properties while imparting some special properties such as hydrophobicity and biodegradability. Seyfi et al. [16] produced silica-containing PU nanocomposite, but they used petroleum-based raw materials. Moreover, SNPs have a huge influence on the increase of the water contact angle. Chen et al. [17] used in situ polymerization for the production of Si-PU nanocomposite and reported that SNPs were evenly distributed in the polymer matrix and also had good physical and mechanical properties, but they also used petroleum-based raw materials. Furthermore, the functionalization of SNP (f-SNP) improves compatibility with polymer matrix. Thus, f-SNP can be used as efficient nanofillers in the fabrication of hydrophobic polymeric nanocomposites. The incorporation of f-SNP with a suitable polymeric material might reveal self-healing properties with adequate mechanical strength and chemical resistance upon strong acid attack. Among the various self-healing polymeric materials, polyurethanes (PU) have been the foremost materials due to their exceptionally versatile properties such as inherent soft-hard segments, high recoverable strain and a broad range of transition temperature of shape recovery [18]. Moreover, PU possesses intrinsic advantages such as adjustable mechanical, thermal, chemical and physical properties owing to the presence of soft and hard segments within the same molecular chain. Furthermore, the globular three-dimensional architecture of hyperbranched PU (HPU) imparts it with exceptional attributes like higher solubility, functionality, reactivity, lower solution and melt viscosity and improved compatibility with other reactants including nanoparticles as compared to their linear analogs. Moreover, bio-based HPU can offer desired biodegradability and biocompatibility as well as sustainability $[18,19]$.

Therefore, an attempt was made to achieve the combined attributes of toughness, shape memory, hydrophobicity and self-healing properties of the in situ fabricated starch-modified HPU nanocomposites using different weight percentages of the f-SNP. The mechanical, thermal and shape memory properties of the fabricated HPU nanocomposites were also evaluated, thereby establishing its structure-property relationship. 


\section{Experimental}

\subsection{Materials}

Fumed Si powder $\left(M_{\mathrm{n}}=60.08 \mathrm{~g} \cdot \mathrm{mol}^{-1}\right.$, Otto chemika biochemika), polyethylene glycol (PEG, $M_{\mathrm{n}}=$ $600 \mathrm{~g} \cdot \mathrm{mol}^{-1}$, Merck, India), sodium dodecyl sulfate $\left(\mathrm{SDS}, M_{\mathrm{n}}=288.37 \mathrm{~g} \cdot \mathrm{mol}^{-1}\right.$, Sigma Aldrich, Germany) and ethanol (Helix, India) were used as received. Soluble starch $\left(M_{\mathrm{n}}=342.30 \mathrm{~g} \cdot \mathrm{mol}^{-1}\right.$, Sigma Aldrich, Germany), polycaprolactone diol with (PCL, $M_{\mathrm{n}}=3000 \mathrm{~g} \cdot \mathrm{mol}^{-1}$, Solvay Co) and 1,4-butanediol (BD, Merck, Germany) were vacuum dried overnight before use. 2,4/2,6 toluene diisocyanate (TDI, Merck, Germany) was used as received. Tetrahydrofuran (THF, SD fine Chem., India) was used after distillation. Xylene (Merck, India) was vacuum distilled and stored using molecular sieves. All other chemicals used in this investigation were of reagent grade. Starch modified hyperbranched polyol (HBSP) was prepared using the same method as reported earlier [18].

\subsection{Preparation of functionalized SNP (f-SNP)} Sodium dodecyl sulfate (SDS) was added to $150 \mathrm{~mL}$ deionized water and stirred magnetically for $30 \mathrm{~min}$. $4 \mathrm{~g}$ silica was added to the above solution. The mass ratio of SDS/silica should not exceed 75 (w/w). The slurry was stirred magnetically at room temperature for around $1 \mathrm{~h}$ to give good dispersion which was followed by 30 minutes of ultrasonication. The slurry was then spray dried on a glass slab. After drying, the particles were collected using a brush. The collected particles were then dried in an oven for $2 \mathrm{~h}$ at $100^{\circ} \mathrm{C}$. The particles were then washed thoroughly with super anhydrous ethanol to remove the unreacted SDS. It was then dried in an oven for $8 \mathrm{~h}$ at $100^{\circ} \mathrm{C}$. To reduce the agglomeration of the SNP, the dried particles were mixed with PEG 600 and stirred mechanically for 5 hours to allow PEG to interact closely with the f-SNP and swell them. To remove the excess PEG, ethanol washing was done using the same protocol as the one used to remove the excess SDS while preparing hydrophobic f-SNP. It was then dried in an oven for 8 hours at $100^{\circ} \mathrm{C}$.

\subsection{Fabrication of HPU nanocomposites}

HPU nanocomposite was prepared via the pre-polymerization technique using a three-necked round-bottomed flask equipped with a nitrogen inlet, a thermometer, a Teflon septum, and a mechanical stirrer.
The required amount of PCL was taken in a reaction flask and dissolved in a desired amount of xylene under constant stirring. DMAc solution of BD was added to it. At room temperature, a desired amount of TDI was added dropwise into the reaction mixture while maintaining the $\mathrm{NCO} / \mathrm{OH}$ at 1 . The reaction was carried out at $70^{\circ} \mathrm{C}$ for $3 \mathrm{~h}$ in a nitrogen atmosphere under constant mechanical agitation to obtain an $-\mathrm{OH}$ terminated pre-polymer. In the second step, HBSP in xylene was introduced into the reaction mixture with the remaining amount of TDI at room temperature (overall $\mathrm{NCO} / \mathrm{OH}=1$ ). The temperature was maintained at $80{ }^{\circ} \mathrm{C}$ followed by addition of f-SNP in xylene and stirred continuously for $4 \mathrm{~h}$ [18]. Three compositions of HPU nanocomposites with $0.5,1$ and 1.5 weight $\%$ of $\mathrm{f}-\mathrm{SNP}$ were fabricated and coded as HPU/SNP0.5, HPU/SNP1, and HPU/ SNP1.5, respectively. Similarly, HPU was also prepared using the same technique without nanomaterial.

\subsection{Characterization techniques}

Fourier transformed infrared (FT-IR) spectra were recorded on a Nicolet (Madison, USA) FT-IR Impact 410 spectrometer in absorbance mode using $\mathrm{KBr}$ pellets. Thermal properties were evaluated using thermogravimetric analysis (TGA) and differential scanning calorimetric (DSC) studies. The thermogravimetric study was carried out by using a PerkinElmer 4000 thermal instrument, in the temperature range of $35-700^{\circ} \mathrm{C}$, at a scanning rate of $10^{\circ} \mathrm{C} \cdot \mathrm{min}^{-1}$, maintaining an inert atmosphere of nitrogen at a gas flow rate of $30 \mathrm{~mL} \cdot \mathrm{min}^{-1}$. Mechanical properties were measured using a Universal Testing Machine (UTM, Zwick Z010, Germany) equipped with a $500 \mathrm{~N}$ load cell operated at a crosshead speed of $50 \mathrm{~mm} \cdot \mathrm{min}^{-1}$ for tensile strength (ASTM D 638) and elongation at break of samples with dimensions $10 \times 1 \times 0.02 \mathrm{~cm}^{3}$. Impact strength is measured in kinetic energy using the formula $1 / 2 \cdot m v^{2}$. A tip or indenter is used to determine the hardness value. The tip or indenter applies force until there is a permanent deformation on the surface. Nanocomposite thin films were mounted on metal place for testing the hardness. The hardness tester was manufactured by Sheen Instruments Ltd, UK. The X-ray diffraction study was carried out at room temperature $\left(25^{\circ} \mathrm{C}\right)$ using a Rigaku X-ray diffractometer (Miniflex, UK) over the range of $2 \theta=$ $10-70^{\circ}$. The water contact angle was measured by a Drop Shape Analyzer (DSA 25, Kruss, Germany). 


\subsection{Sessile water drop test}

Sessile water drop test technique is widely used to measure the surface energy of a solid surface. It can also be used to measure the contact angle of water with a solid surface. A water drop is dropped into the surface, and the contact angle $\left(\theta\left[{ }^{\circ}\right]\right)$ is measured which determines if the surface is hydrophilic, hydrophobic or super-hydrophobic. The water contact angle is the angle between the solid surface and the tangent drawn from the point where liquid-vapour interface meets the solid.

\subsection{Shape memory test}

The ability of the shape memory polymers to remember their initial form under the action of a suitable stimulus is very important. They have wide applications in industries such as construction and automobile. The test was carried out using thin rectangular samples of $3 \times 0.5 \mathrm{~cm}^{2}$. The samples were heated at $60^{\circ} \mathrm{C}$ and stretched to twice the original length. The stretched samples were immersed in an ice bath at $-(15 \pm 5)^{\circ} \mathrm{C}$ for $5 \mathrm{~min}$ to to fix their shape. Subsequently, they were reheated and the time taken to regain the original shape was recorded. The fixity and recovery $\%$ of the samples were then calculated using Equations (1) and (2):

Fixity $[\%]=\frac{I_{2}-I_{0}}{I_{0}} \cdot 100$

Recovery $[\%]=\frac{I_{1}-I_{3}}{I_{0}} \cdot 100$

where, $l_{0}$ is the original length; $l_{1}$ the stretched length; $l$ the cooled length and $l_{3}$ the length after reheating.

\subsection{Self-healing test}

Thin strips of the nanocomposites were taken, to which a cut of $5 \mathrm{~mm}$ length and $0.5 \mu \mathrm{m}$ depth was made with a razor blade in the transverse direction. The self-healing performance was studied under microwave of $360 \mathrm{~W}$, by noting the times taken to heal the crack.

\subsection{Biodegradation test}

Biodegradation study was done by McFarland turbidity method using Pseudomonas aeruginosa (P. aeruginosa) and Bacillus subtilis (B. subtilis) as the bacterial strain $[18,20]$. A medium of mineral salts containing $2.0 \mathrm{~g}$ of $\left(\mathrm{NH}_{4}\right)_{2} \mathrm{SO}_{4}, 2.0 \mathrm{~g}$ of $\mathrm{Na}_{2} \mathrm{HPO}_{4}$, $4.75 \mathrm{~g}$ of $\mathrm{KH}_{2} \mathrm{PO}_{4}, 1.2 \mathrm{~g}$ of $\mathrm{MgSO}_{4} \cdot 7 \mathrm{H}_{2} \mathrm{O}, 0.5 \mathrm{mg}$ of
$\mathrm{CaCl}_{2} \cdot 2 \mathrm{H}_{2} \mathrm{O}, 100 \mathrm{mg}$ of $\mathrm{MnSO}_{4} \cdot 5 \mathrm{H}_{2} \mathrm{O}, 70 \mathrm{mg}$ of $\mathrm{ZnSO}_{4} \cdot 7 \mathrm{H}_{2} \mathrm{O}, 10 \mathrm{mg}$ of $\mathrm{H}_{3} \mathrm{BO}_{3} \cdot 5 \mathrm{H}_{2} \mathrm{O}, 100 \mathrm{mg}$ of $\mathrm{CuSO}_{4} \cdot 7 \mathrm{H}_{2} \mathrm{O}, 1 \mathrm{mg}$ of $\mathrm{FeSO}_{4} \cdot 7 \mathrm{H}_{2} \mathrm{O}$, and $10 \mathrm{mg}$ of $\mathrm{MoO}_{3}$, all in $1.0 \mathrm{~L}$ of demineralized water, was prepared. It was sterilized for $15 \mathrm{~min}$ at $120^{\circ} \mathrm{C}$ under a pressure of $15 \mathrm{lb}$ and then allowed to cool to room temperature. The bacterial strain of $P$. aeruginosa and B. subtilis were cultured in the medium inside an incubator shaker at $37^{\circ} \mathrm{C}$ for $48 \mathrm{~h} .100 \mu \mathrm{L}(108 \mathrm{mi}-$ crobes $/ \mathrm{mL}$, as calculated by McFarland turbidity method) of the cultured medium was taken in a conical flask containing $10 \mathrm{~mL}$ of the prepared salt medium. The polymeric samples were sterilized by autoclaving and were incubated inside the medium under sterile conditions at $37^{\circ} \mathrm{C}$. The flasks containing mineral salt medium but without any bacterial strain was used as the control. The extent of biodegradation was studied from the measurement of weight loss [\%] of the degraded HPU nanocomposite films, measured after 5 weeks of exposure to the bacterial strains, based on the Equation (3):

$W=\frac{W_{0}-W_{\mathrm{t}}}{W_{\mathrm{t}}} \cdot 100$

where $W_{0}$ and $W_{\mathrm{t}}$ are the weights of the sample before and after degradation, respectively, at the time of interest, $t$. The bacterial growth is indicated by increase in turbidity of the medium with time. The optical density (OD) of the microorganism was monitored by measuring the absorbance of the medium at $600 \mathrm{~nm}$ with respect to the control. The experiment was performed in triplicate.

\section{Results and discussion}

\subsection{Characterization of f-SNP}

The FTIR spectrum (Figure 1a) of f-SNP showed the characteristic peaks for silica at 795 and $957 \mathrm{~cm}^{-1}$, attributed to $\mathrm{Si}-\mathrm{O}-\mathrm{Si}$ symmetric stretching and $\mathrm{Si}-\mathrm{OH}$ stretching, respectively. The peak at $1638 \mathrm{~cm}^{-1}$ is due to $\mathrm{O}-\mathrm{H}$ bending vibration mode of physisorbed water molecules. The characteristic absorption bands of the stretching vibrations of the $\mathrm{H}$-bonded $\mathrm{Si}-\mathrm{OH}$ and $-\mathrm{OH}$ of the physisorbed water molecules were integrated into a broad peak at $3436 \mathrm{~cm}^{-1}$. The appearance of a peak at $2918 \mathrm{~cm}^{-1}$ showed the presence of alkyl chain present in the SDS functionalized silica nanoparticles. The $\mathrm{Si}-\mathrm{H}$ band was not detected in the FTIR spectrum due to its low concentration [21]. The very intense broad band at $1100 \mathrm{~cm}^{-1}$ can be assigned to the transversal optical (TO) 


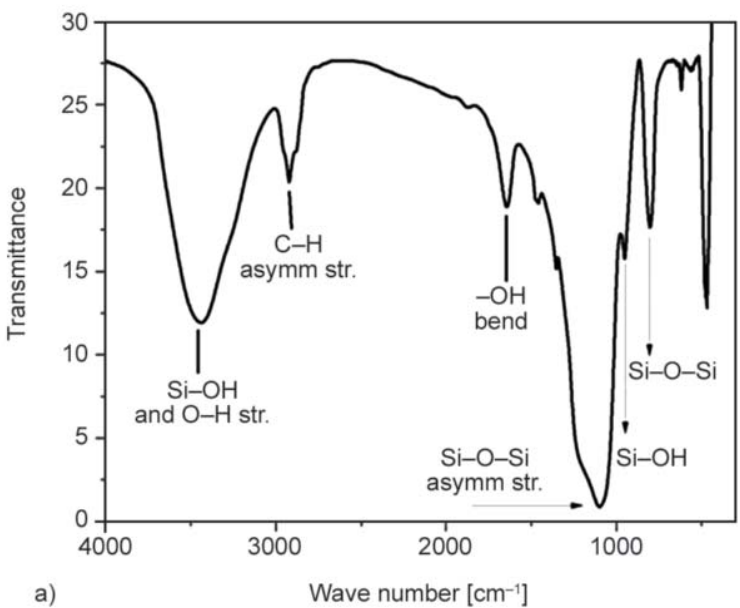

Figure 1. (a) FTIR spectrum and (b) XRD pattern of f-SNP.

modes of the $\mathrm{Si}-\mathrm{O}-\mathrm{Si}$ asymmetric stretching vibrations [22].

Figure $1 \mathrm{~b}$ shows the XRD pattern of f-SNP which confirms the powder amorphous silica nanoparticles. The highest peak at $22.7^{\circ}$ confirms the presence of $\mathrm{f}-\mathrm{SNP}$ in the sample, with a wide range of angle $2 \theta=22$ to $24^{\circ}$ [23]. The broadness of the peak also indicates the amorphous nature of $\mathrm{SiO}_{2}$. The

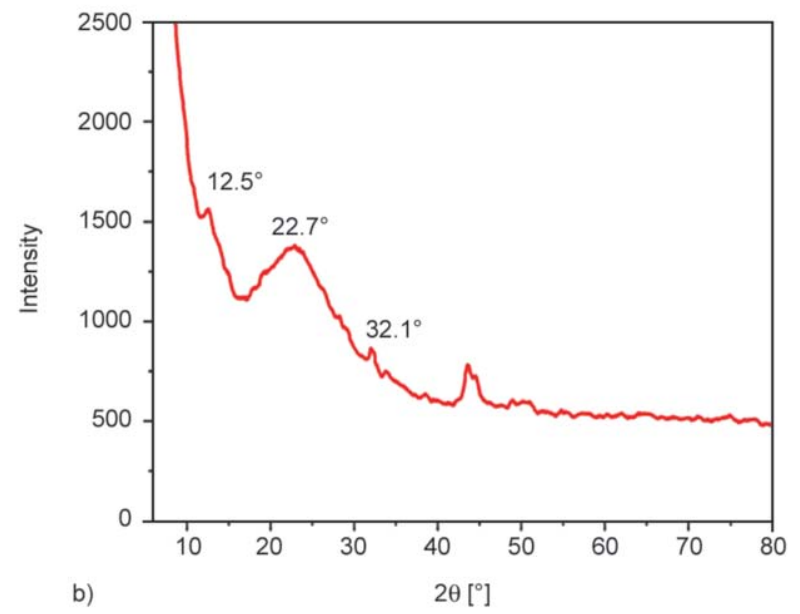

small peak at around $2 \theta$ values of 12.5 and $32.1^{\circ}$ are due to the organic moieties, PEG and SDS, respectively $[24,25]$.

The TEM images (Figure 2a-2e) show that f-SNP is amorphous in nature with no fringes at $5 \mathrm{~nm}$ resolution. Also Selected Area Electron Diffraction (SAED) (Figure 2f) pattern shows no spots indicating the amorphous nature of the f-SNP. The shape is

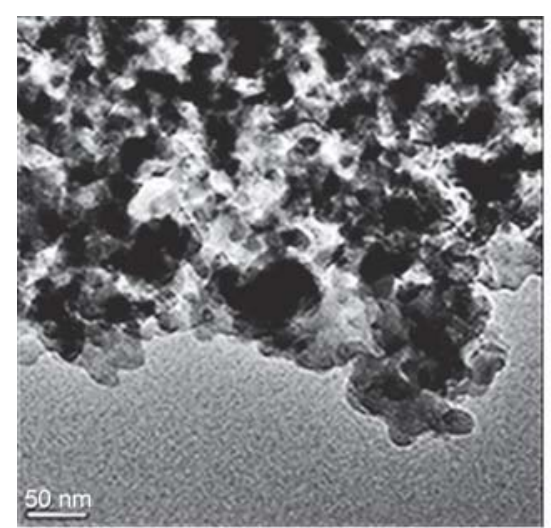

a)

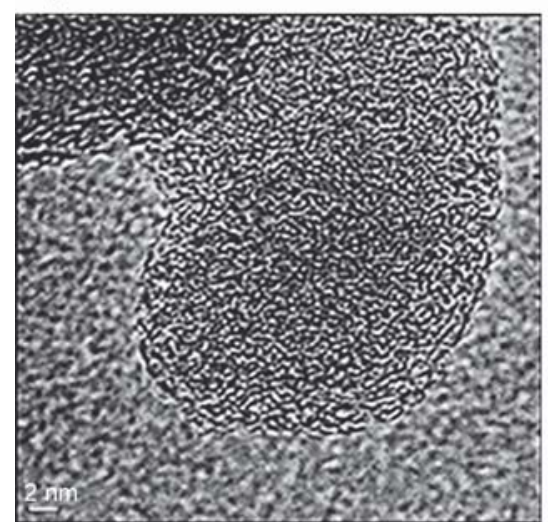

d)

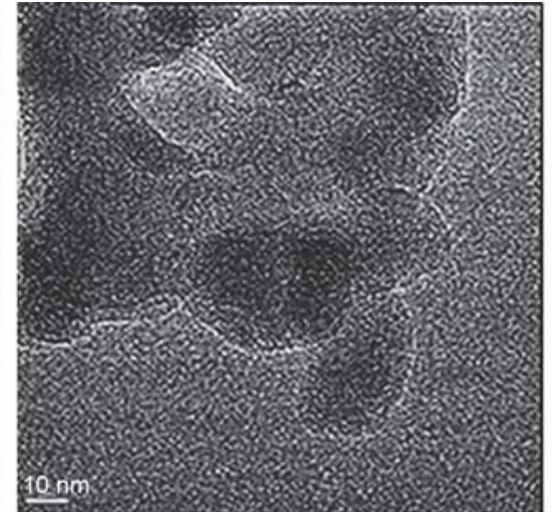

b)

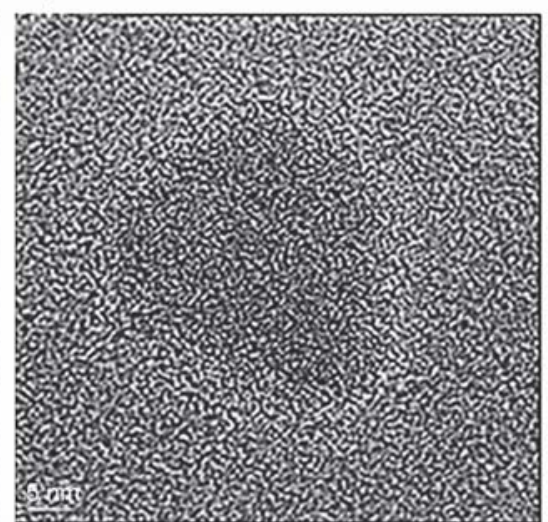

e)

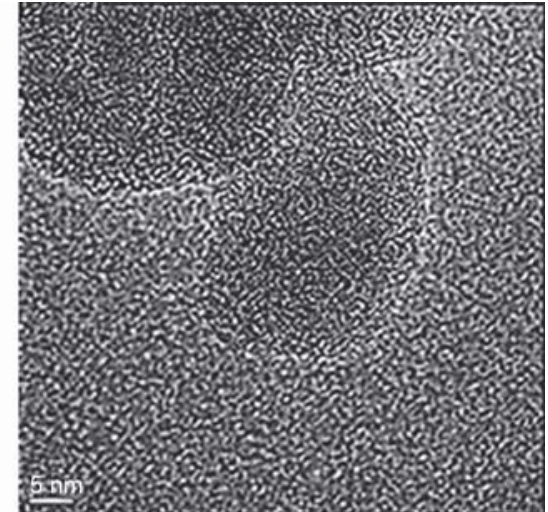

c)

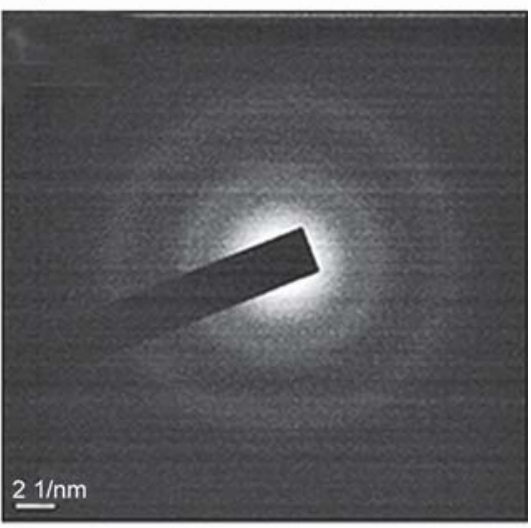

f)

Figure 2. TEM image showing agglomeration of f-SNP at (a) $50 \mathrm{~nm}$ magnification. (b) Zoomed in TEM image of the agglomerated f-SNP at $10 \mathrm{~nm}$ magnification. (c) and (d) Zoomed-in image of a single f-SNP from the agglomerate at $5 \mathrm{~nm}$ and $2 \mathrm{~nm}$ magnification respectively. (e) TEM image of an isolated f-SNP at $5 \mathrm{~nm}$ magnification. (f) SAED pattern of f-SNP. 
irregular even though some particles show spherical or elliptical shapes. There is no particular order of arrangement for f-SNP. The distribution can be described as clustered. The sizes of f-SNP are between (23-26) nm.

\subsection{Fabrication and characterization of HPU nanocomposites}

The fabrication of HPU nanocomposites was done by an in situ polymerization technique (Figure 3). The basic features for the successful preparation of nanocomposite are concentration of the reactants, nature, and amount of nanomaterial, rate of addition of the branching moiety, time and temperature of the reaction [18]. Consequently, branching unit was added drop-wise at room temperature in a dilute xylene solution (15-20\%) to avoid gel formation, in the second step of the reaction. Also, the nanohybrid was slowly incorporated in the second step to attain strong interfacial interactions with the HPU matrix. Strong interfacial interactions result in better stabilization of nanohybrid within the matrix.

The FTIR spectra (Figure 4a) of HPU and HPU/SNP1 reveal the absence of peaks for free $-\mathrm{NCO}$ at $2270 \mathrm{~cm}^{-1}$ indicates that the reaction was completed. The peaks at $3325-3346 \mathrm{~cm}^{-1}$ represent the overlapping of $-\mathrm{NH}$ and $-\mathrm{OH}$ stretching vibrations in the polymers. Two peaks between the range of 2934-2948 and 2849-2864 $\mathrm{cm}^{-1}$ are because of symmetric and asymmetric vibration of $-\mathrm{CH}_{2}$ respectively.
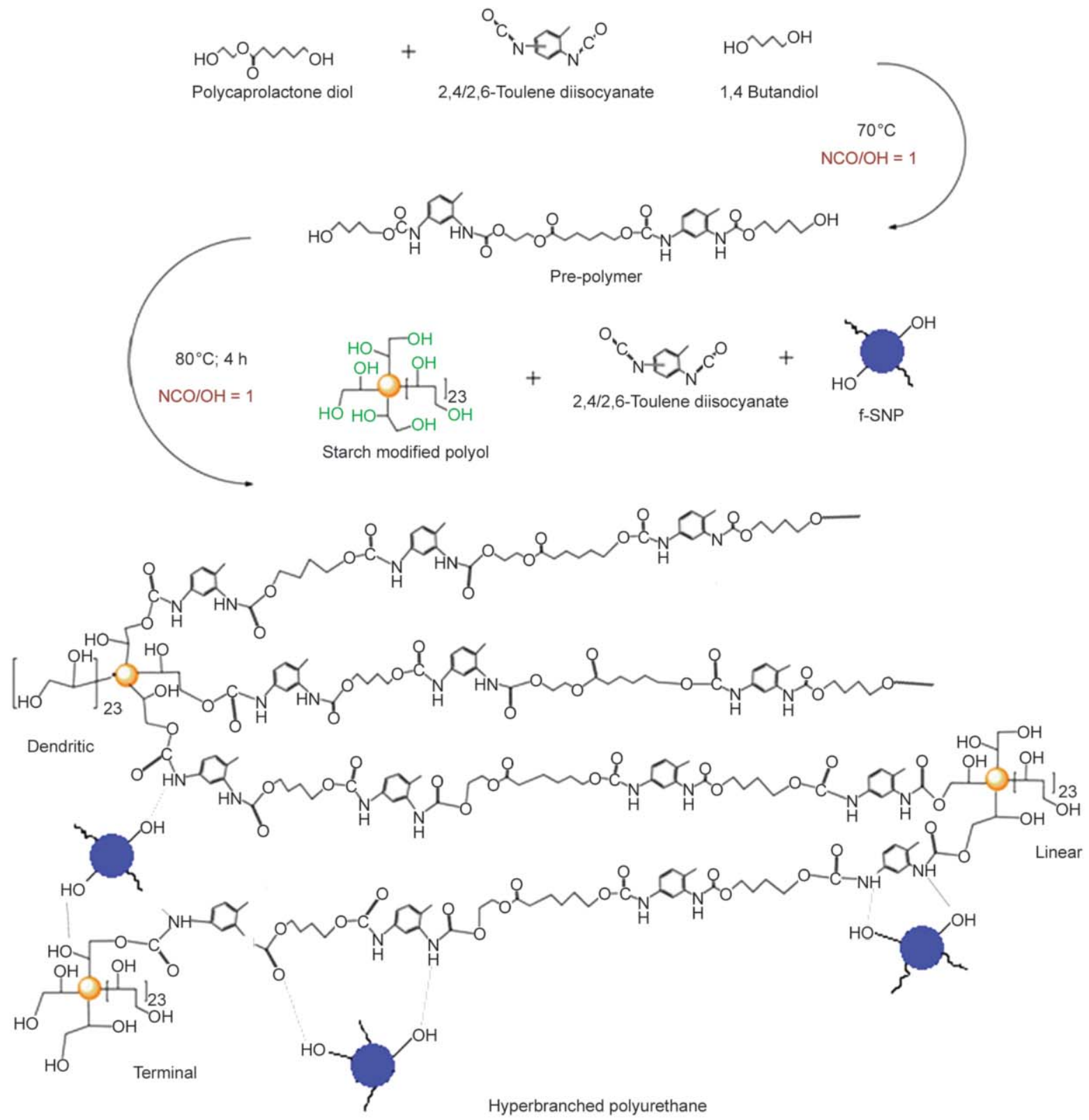

Figure 3. Fabrication of HPU nanocomposite. 

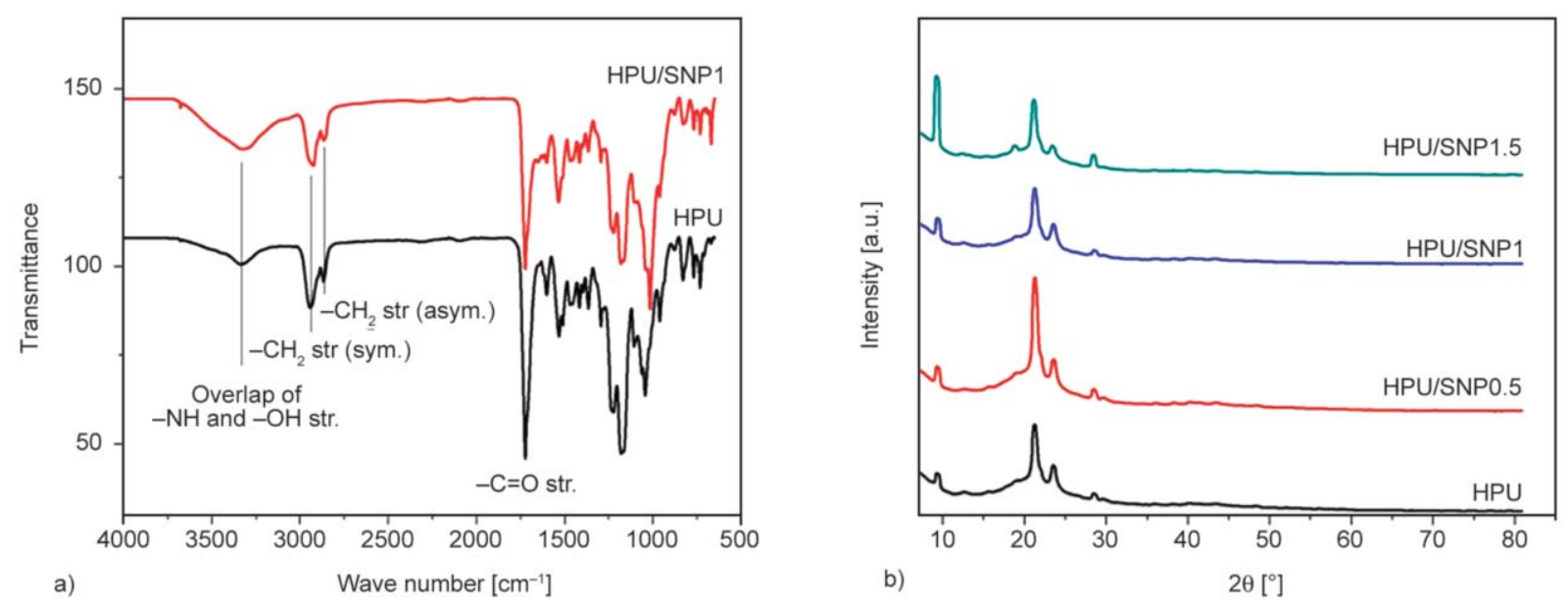

Figure 4. (a) FTIR spectra of HPU and its nanocomposites; and (b) XRD patterns of HPU and its nanocomposites.

The peaks in the range of $1716-1722 \mathrm{~cm}^{-1}$ represent $-\mathrm{C}=\mathrm{O}$ stretching vibrations. The peaks between $1518-11530 \mathrm{~cm}^{-1}$ are because of $-\mathrm{C}-\mathrm{N}$ stretching/ $-\mathrm{N}-\mathrm{H}$ vibrations while the peaks in the range of $1042-1046 \mathrm{~cm}^{-1}$ represent $-\mathrm{O}-\mathrm{C}=\mathrm{O}$ stretching vibration of urethane $[18,19]$.

The XRD curves (Figure 4b) of the nanocomposites reveal two distinct peaks in the range of 21.1 and $22^{\circ}$ due to the presence of crystalline PCL moieties in the HPU structure $[18,19]$. The broad peak at $22.1^{\circ}$ due to $\mathrm{SiO}_{2}$ is masked by the peaks of PCL moieties. The peak at $12.5^{\circ}$ can be because of the remaining $-\mathrm{OH}$ residues from the ethanol washing procedure. The peak at around $31.8^{\circ}$ can be attributed to SDS, which was used to swell the nanoparticles [24, 25]. From the TEM images (Figure 5) of HPU/SNP, we can conclude that the as the dispersed f-SNP are amorphous in nature as no fringes occur at $5 \mathrm{~nm}$ resolution and the SAED pattern shows no spots. The dispersion of nanoparticles in the HPU matrix is not uniform, and clusters can be seen even though some individual particles of size $19-20 \mathrm{~nm}$ can be seen in the HPU matrix.

\subsection{Mechanical properties}

The mechanical properties of HPU and its nanocomposites are tabulated in Table 1. Stress $v s$ strain curve makes it evident that upon incorporation of f-SNP in the HPU polymer matrix the tensile strength and elongation at break significantly increased (Figure 6). It is observed that HPU/SNP1.5 exhibited comparatively similar tensile strength which is attributed to the fact f-SNP particles were homogeneously dispersed in the HPU/SNP1 and HPU/SNP1.5 matrices. The f-SNP contains polar functionalities that can interact with the HPU matrix through secondary interactions or covalently. These strong interactions stiffen the hard segments of HPU and facilitate the successful load transfer from HPU matrix to the nanomaterial in the nanocomposite [19]. Further, the highest elongation at break and remarkable tensile strength in $1 \mathrm{wt} \% \mathrm{f}-\mathrm{SNP}$ confers it with the highest toughness. However, there is a decrease of tensile strength and elongation at break values upon incorporation of $2 \mathrm{wt} \% \mathrm{f}-\mathrm{SNP}$ in the HPU matrix, mainly attributed to the agglomeration of f-SNP within the matrix. HPU/SNP1 exhibits the highest elongation at break and toughness among the others. This is due to incorporation of $1 \mathrm{wt} \%$ of $\mathrm{f}-\mathrm{SNP}$ into the HPU matrix which proves to be the optimum dose level to provide good compatibility between HPU and f-SNP, excellent dispersion and strong interaction of the nanomaterial with the polymer matrix. The simultaneous enhancement of tensile strength and elongation at break resulted in the highest toughness for HPU/SNP1 nanocomposite. This optimum loading of the nanomaterial aligns the polymeric chains in the early stages of tensile loading, which allows them to orient the nanomaterial along the direction of loading, thus enhancing the elongation at break to the maximum. It is evident from the table that incorporation of f-SNP into the HPU matrix increases the scratch resistance. The reason for increase of scratch resistance can be credited to elastic-plastic transition effect of f-SNP, which is the ability of certain nanoparticles to impart plastic behavior into the matrix upon incorporation of the nanoparticles [26]. Again, with increase in the amount of f-SNP, the hardness of the HPU nanocomposites increases. All of the HPU nanocomposites displayed excellent impact 


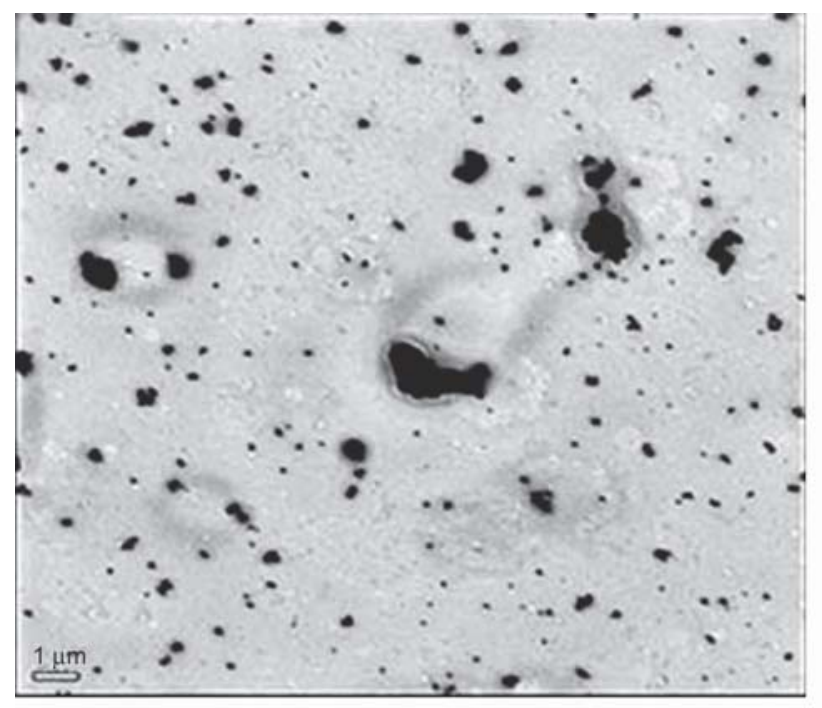

a)

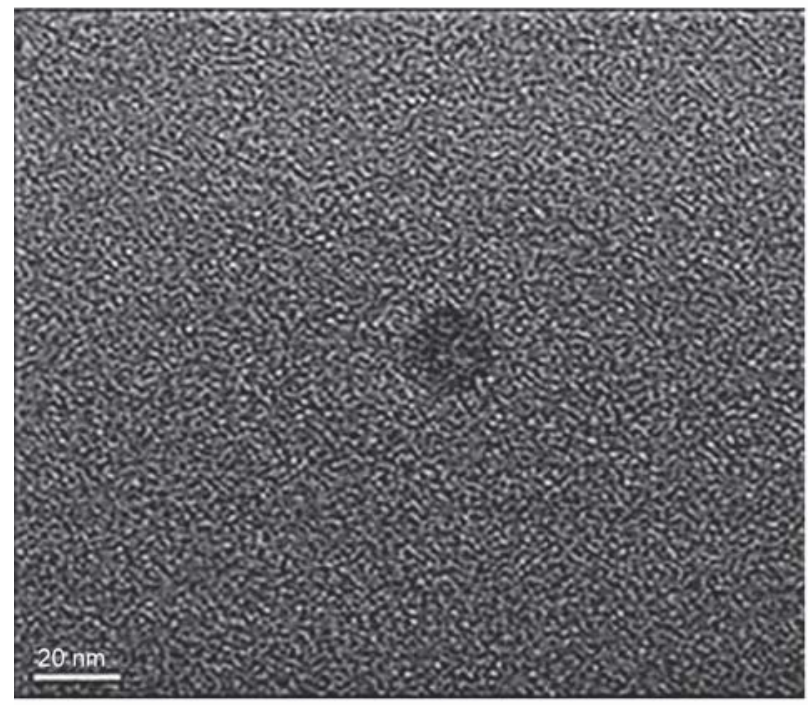

c)

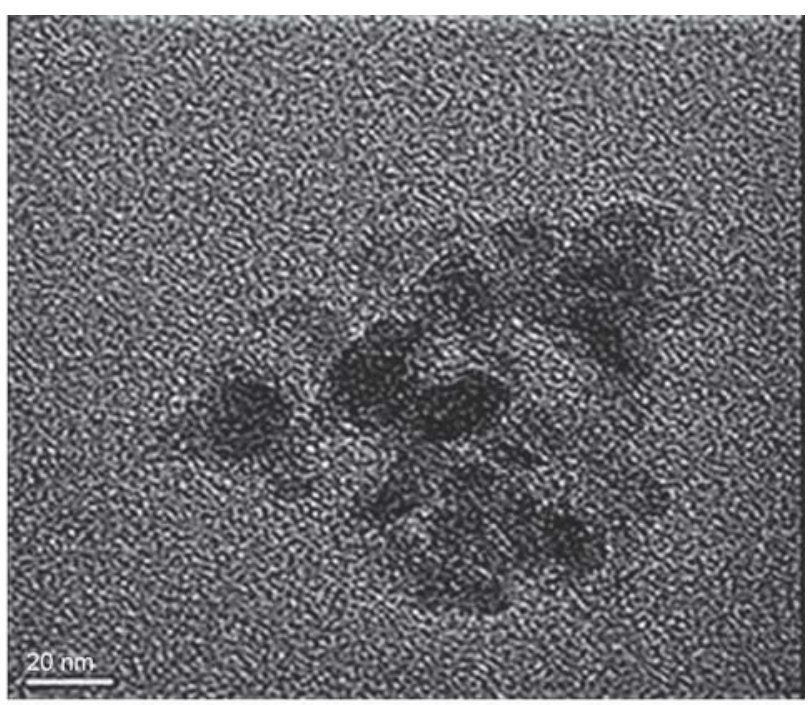

b)

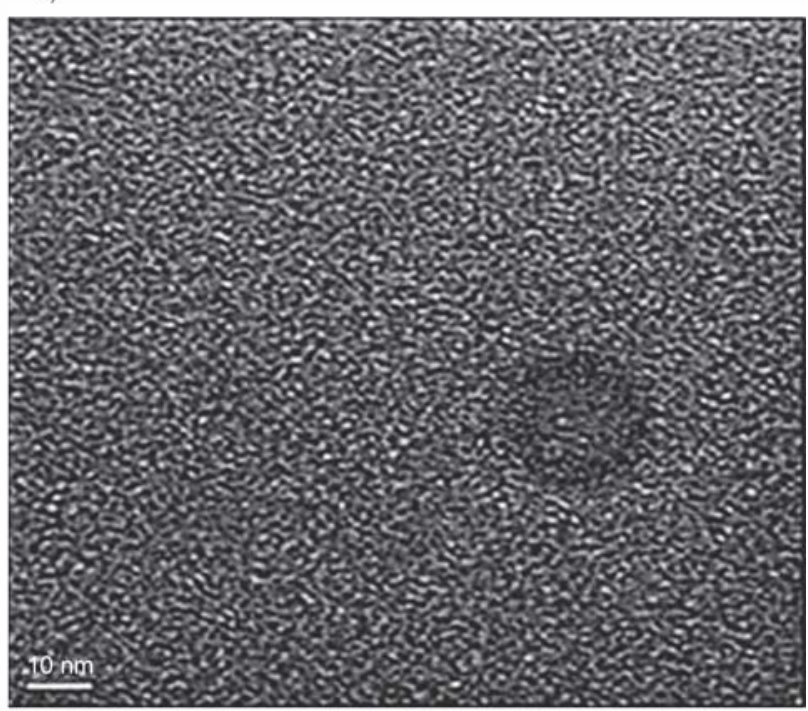

d)

Figure 5. (a) TEM image showing agglomeration of f-SNP at $1 \mu \mathrm{m}$ magnification. (b) Zoomed in TEM image of the agglomerate at $20 \mathrm{~nm}$ magnification. (c) and (d) TEM image of a single f-SNP at $20 \mathrm{~nm}$ and $10 \mathrm{~nm}$ magnification, respectively.

Table 1. Properties of HPU and its nanocomposites.

\begin{tabular}{|c|c|c|c|c|c|c|}
\hline \multicolumn{2}{|c|}{ Property } & HPU & HPU/SNP0.5 & HPU/SNP1 & HPU/SNP1.5 & HPU/SNP2 \\
\hline Tensile strength & {$[\mathrm{MPa}]$} & $17.0 \pm 0.5$ & $18.56 \pm 0.2$ & $23.6 \pm 0.4$ & $25.8 \pm 0.2$ & $14.0 \pm 0.2$ \\
\hline Elongation at break & [\%] & $1056 \pm 4$ & $1295 \pm 2$ & $1495 \pm 2$ & $1192 \pm 4$ & $1315 \pm 5$ \\
\hline Scratch hardness & {$[\mathrm{kg}]$} & $6.5 \pm 0.4$ & $7.0 \pm 0.3$ & $7.5 \pm 0.3$ & $7.5 \pm 0.2$ & $7.5 \pm 0.5$ \\
\hline Impact strength $^{\mathrm{a}}$ & {$\left[\mathrm{kJ} \cdot \mathrm{m}^{-1}\right]$} & $>19.02$ & $>19.02$ & $>19.02$ & $>19.02$ & $>19.02$ \\
\hline Hardness & [Shore A] & 45 & 68 & 75 & 72 & 72 \\
\hline Toughness $^{\mathrm{b}}$ & {$\left[\mathrm{MJ} \cdot \mathrm{m}^{-3}\right]$} & $136.73 \pm 4$ & $204.90 \pm 2$ & $340.88 \pm 3$ & $211.31 \pm 2$ & $166.34 \pm 3$ \\
\hline Advancing angle & {$\left[{ }^{\circ}\right]$} & 76.7 & 101.4 & 104.4 & 105.2 & - \\
\hline Receding angle & {$\left[{ }^{\circ}\right]$} & 75.2 & 99 & 101.4 & 101.7 & - \\
\hline Angle of hysteresis & {$\left[{ }^{\circ}\right]$} & 1.5 & 2.4 & 3.0 & 3.5 & - \\
\hline Shape fixity & {$[\%]$} & 90.0 & 97.5 & 98.6 & 99.3 & - \\
\hline Shape recovery & {$[\%]$} & 92.0 & 97.6 & 98.6 & 99.4 & - \\
\hline
\end{tabular}

${ }^{a}$ Maximum limit of the instrument for impact strength was $19.02 \mathrm{~kJ} \cdot \mathrm{m}^{-1}$ (highest).

${ }^{\mathrm{b}}$ Toughness was calculated by integrating the area under stress-strain curves. 


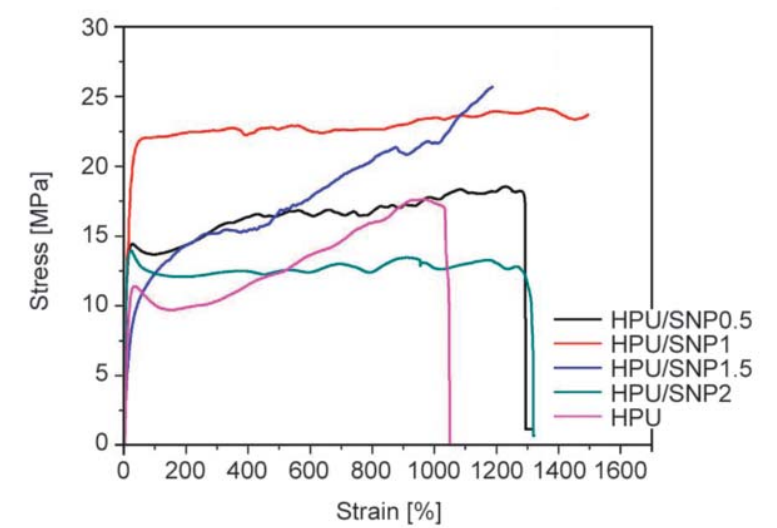

Figure 6. Stress $v s$. strain curves for HPU and its nanocomposites.

strength of $19.02 \mathrm{~kJ} \cdot \mathrm{m}^{-1}$ (maximum limit of the used instrument), due to good polymer chain flexibility. The increase in the value of impact strength can be explained by the ability of nanoparticles to form a tortuous pathway and the ability to act as a reducer for crack propagation. They also help in better load transfer from the matrix and act as links between the HPU chains.

\subsection{Thermal properties}

TGA thermograms of HPU nanocomposites display two-step degradation patterns owing to the presence of both hard and soft segments in the HPU matrix (Figure 7). This signifies that the loading and nature of the nanomaterial do not significantly determine the degradation pattern of the nanocomposites. The nanocomposites displayed higher peak temperatures for second step degradation, upon increase in loading of f-SNP (Table 2) which is due to good dispersibility of SNP within the HPU matrix. The f-SNP acts as the thermal stabilizer and capably restricts the chain mobility.

Further, f-SNP contributes in the physical cross-linking process by virtue of its functional groups which result in making the degradation process highly energy consuming and thus provide superior thermostability to the nanocomposites. The enhanced barrier properties of the nanocomposites also help in retaining the produced volatiles during the decomposition process in the polymer matrix $[27,28]$.

\subsection{Determination of water contact angle}

The hydrophobicity of HPU surface is based upon surface geometrical microstructure (roughness) and surface chemical composition (intrinsic hydrophobicity) and the surface geometrical microstructures (roughness) [29]. The inherent hydrophobic character of HPU nanocomposite is provided by the nonpolar moieties such as long aliphatic hydrocarbon chains of HBSP and aliphatic and aromatic parts of diisocyanates. Additionally, the low surface energy and non-polar nature of f-SNP also significantly contribute to enhancing the hydrophobicity as seen in the digital images of water droplets of HPU surfaces

Table 2. Thermal properties of HPU and its nanocomposites.

\begin{tabular}{|ll|c|c|c|}
\hline \multicolumn{2}{|c|}{ Parameter } & HPU/SNP0.5 & HPU/SNP1 & HPU/SNP1.5 \\
\hline Peak Temp for $1^{\text {st }}$ degradation & {$\left[{ }^{\circ} \mathrm{C}\right]$} & 335 & 327 & 323 \\
\hline Peak Temp for $2^{\text {nd }}$ degradation & {$\left[{ }^{\circ} \mathrm{C}\right]$} & 400 & 405 & 411 \\
\hline Weight residue & {$[\%]$} & 8.3 & 11.2 & 5.8 \\
\hline
\end{tabular}
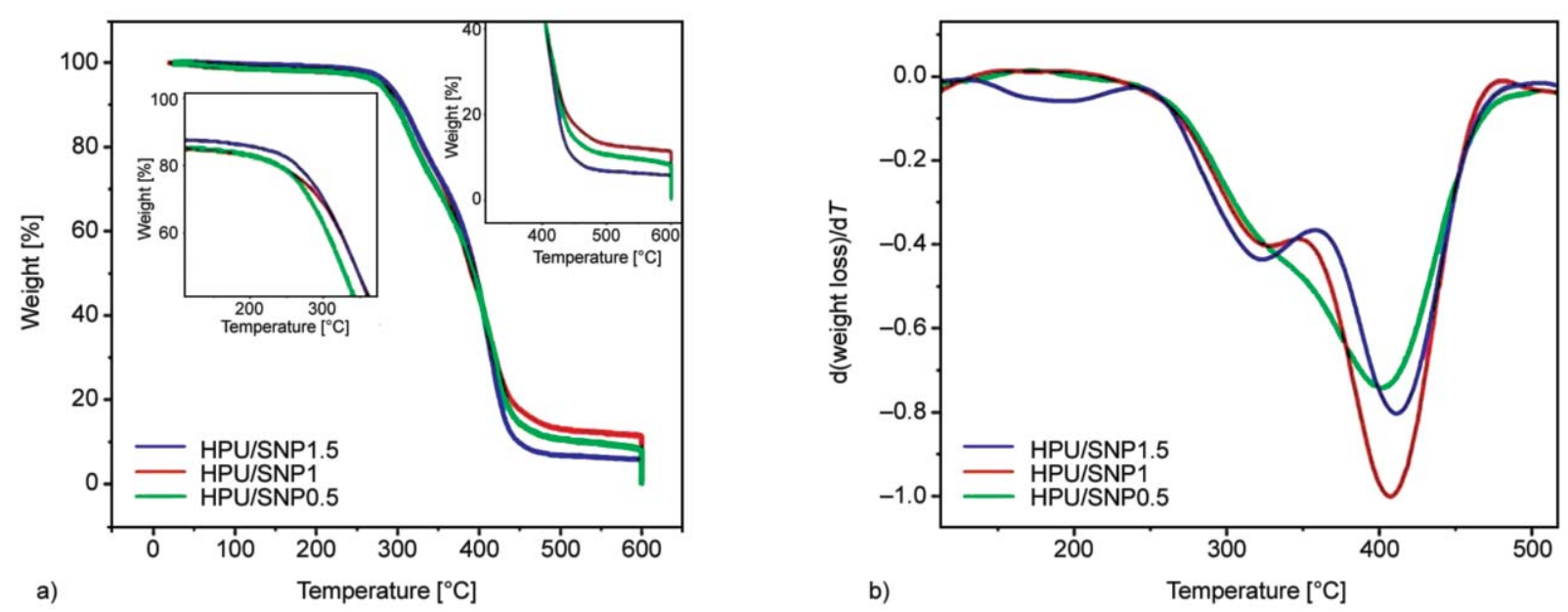

Figure 7. (a) TGA thermograms and (b) DTG curves of HPU and its nanocomposites. 


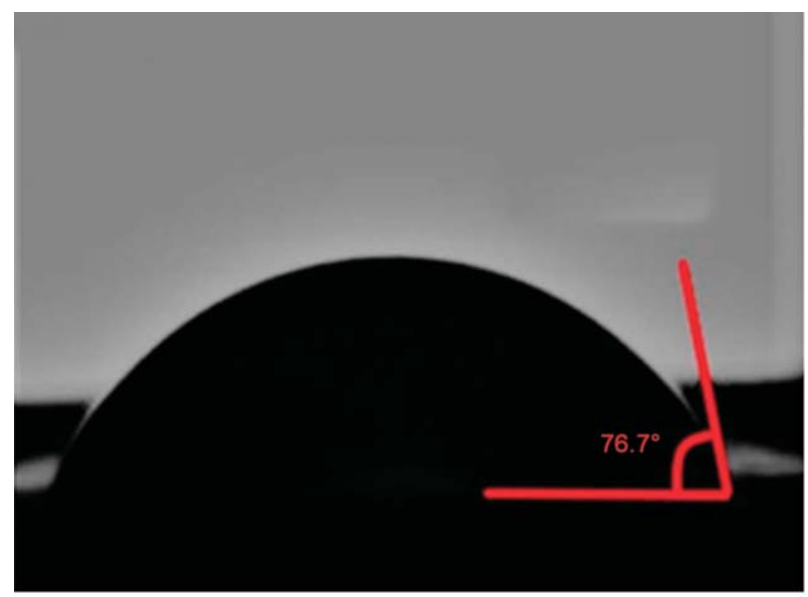

a)

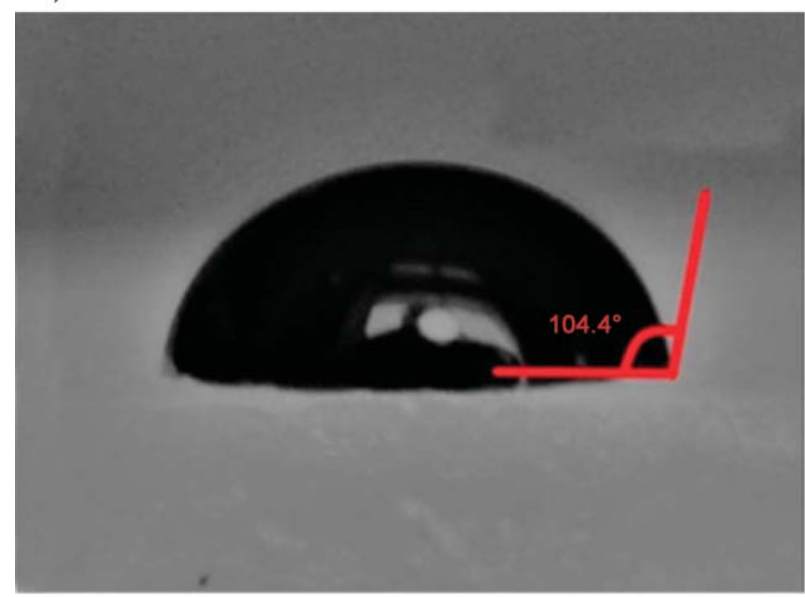

c)

Figure 8. Water contact angles (a) HPU, (b) HPU/SNP0.5, (c) HPU/SNP1, and (d) HPU/SNP1.5.
(Figure 8). Moreover, the incompatibility of amorphous f-SNP with the crystalline PCL moiety in the structure of HPU creates microroughness on the HPU surface. The combination of these two factors results in the hydrophobicity of the surface [30]. It is observed that the contact angle increases with increase in the f-SNP content, as well as due to the enhanced incompatibility from HPU/SNP0.5 to HPU/SNP1 (Table 1). Again, angle of hysteresis (difference between advancing angle and receding angle) determines the rolling ability of the water droplets on the surface of HPU. The lower value of the angle of hysteresis represents higher hydrophobicity and hence, better self-cleaning effect [31]. From Table 1 it is evident that upon incorporation of f-SNP, the water contact angle of the nanocomposites increases.

\subsection{Shape memory behavior}

The non-contact triggered thermoresponsive shape memory behavior of the nanocomposites is shown in Figure 9. HPU and its nanocomposites exhibited remarkable shape fixity and recovery under the thermal

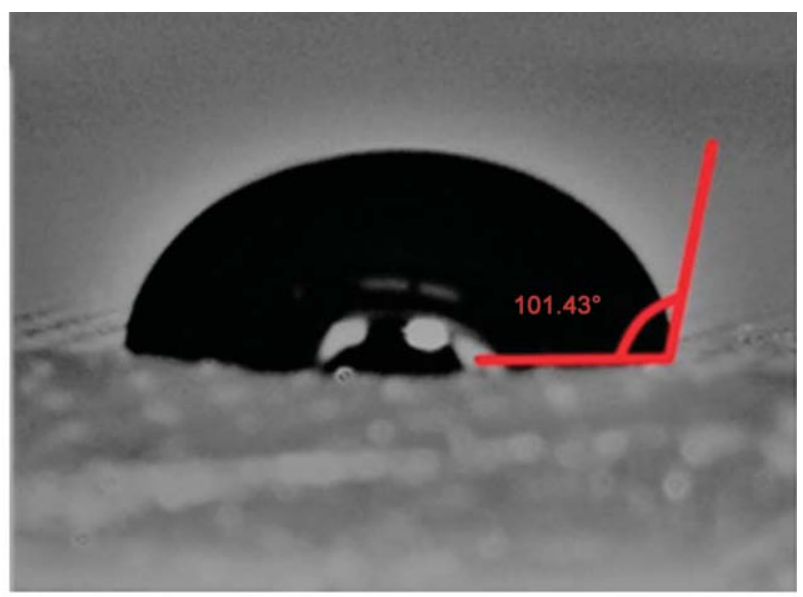

b)

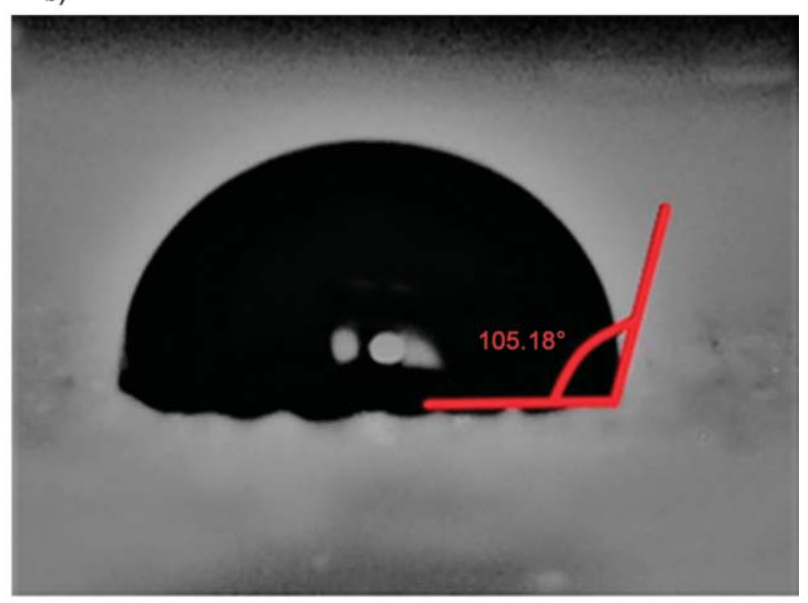

d) energy due to good heat capacity of f-SNP. In the permanent shapes of HPU and its nanocomposites, the polymeric chains are oriented in a random coil arrangement i.e., at their maximum entropic state [18]. On heating the films, their chain mobility is activated owing to which they can be stretched easily to twice their length. Subsequently, the stretched films are quenched $-(15 \pm 5)^{\circ} \mathrm{C}$ for $5 \mathrm{~min}$. This results in fixing the temporary stretched shape by kinetic freezing of the polymeric chains and thus, minimizes their entropic state. On removing the mechanical stress, the polymeric chains do not have adequate energy to revert to their original shape. Upon reheating the films at $60^{\circ} \mathrm{C}$, the molecular chain mobility is restored and gains entropy to return to random coil motion. The shape recovery of HPU nanocomposites was found to be better as compared to HPU due to the increased stored energy of system. This is attributed to multifunctional moiety, hyperbranched polyol, homogeneous distribution of hard segments, increased secondary interactions in the structure of HPU matrix and homogenization of reinforcing 


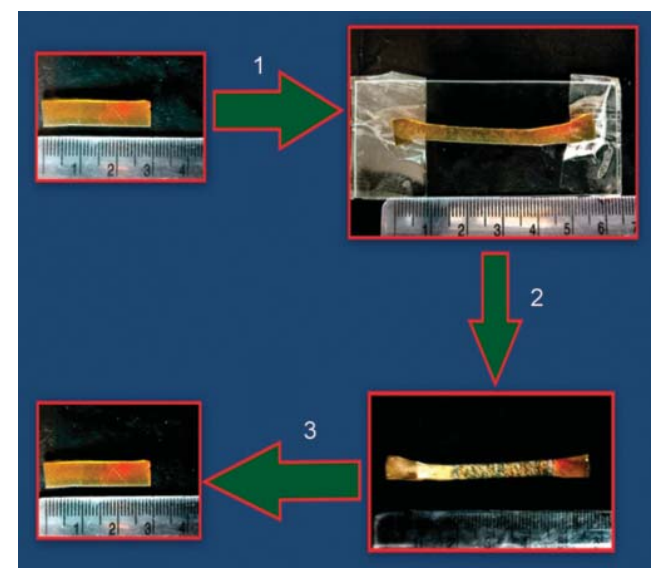

Figure 9. Shape memory behavior of HPU/SNP nanocomposite.

nanomaterial, f-SNP $[18,19]$. The shape fixity and shape recovery of the nanocomposites were found to be enhanced with increase in the weight $\%$ of the nanomaterial (Table 1). There was increased stored energy of the uniformly distributed f-SNP which enhanced its interaction with the hard segment of HPU by generation of strong net points. Thus, f-SNP helped the nanocomposites in achieving high recovery stress due to the release of the stored elastic strain. Also, f-SNP created a huge amount of stored elastic strain energy due to the presence of strong interfacial interactions with the HPU matrix $[32,33]$. Thus, by deforming such thermo-responsive polymers above their $T_{\mathrm{m}}$, the desired shape can be attained which can be sustained after cooling.

\subsection{Self-healing behavior}

The self-healing behavior of HPU and its nanocomposites was studied by optical imaging (Figure 10). The fracture of the nanocomposite was effectively healed by exposure to $M W$ power input of $360 \mathrm{~W}$. The optical images prior to and after the healing process show the complete healing of the nanocomposite films. During the healing process, the absorbed MW energy by f-SNP results in the oscillation of its dipoles, thereby generating heat due to friction at the nanohybrid-polymer interface [34]. The nanomaterial efficiently transfers the absorbed energy to the polymeric matrix that melts the soft segment and initiates molecular diffusion (Brownian movement) of the segment towards the crack. The diffusion and rearrangement of the soft segment help to achieve repair of the damaged site [35]. On the other hand, the sample dimensions of HPU are retained by its hard

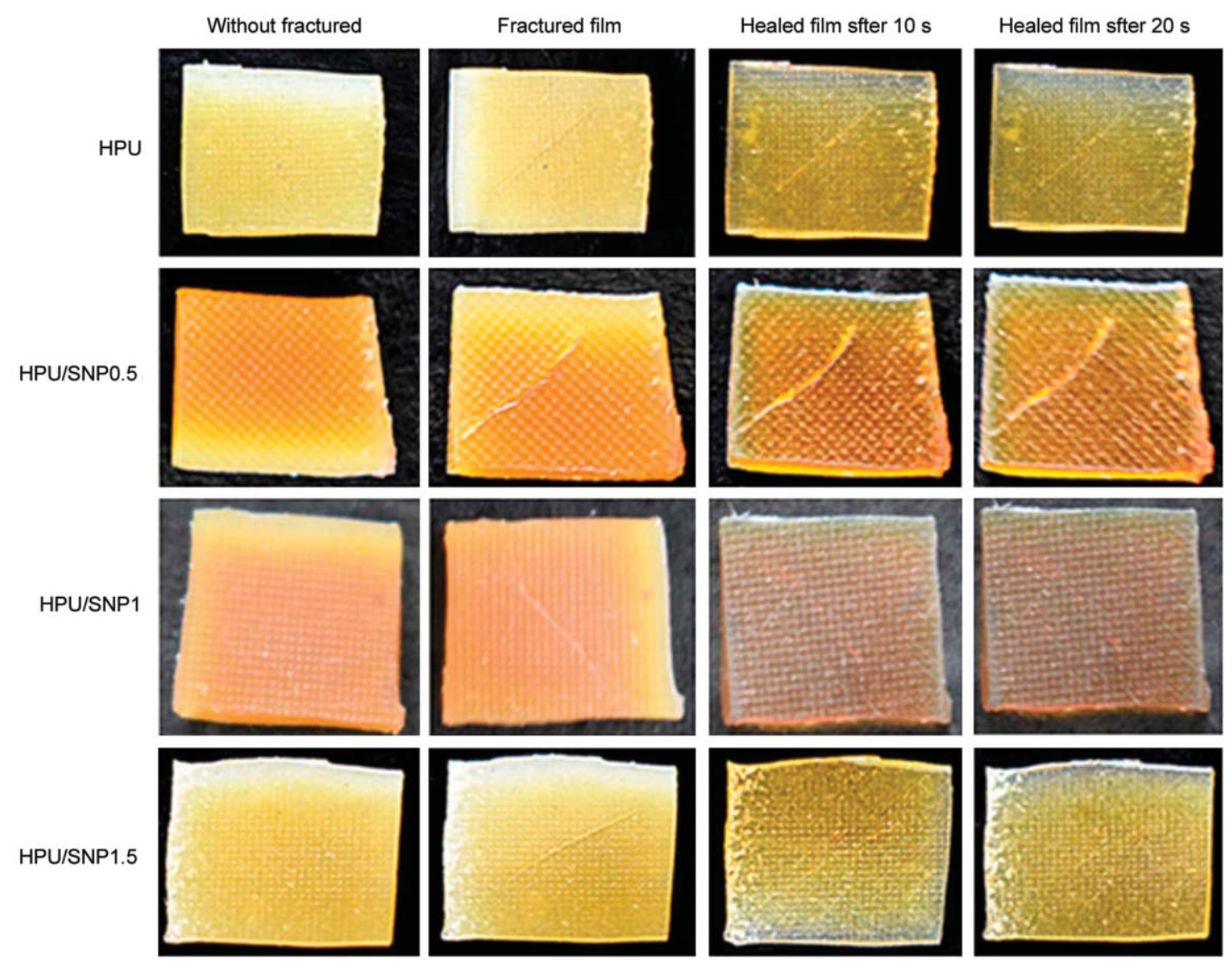

Figure 10. Self-healing behavior of HPU and its nanocomposites. 
segment of HPU. Further, the study also demonstrated that the used energy of the stimulus was inadequate to cause any dimensional degradation or instability as established by TGA analysis of the nanocomposites. Apposite variation of the nanomaterial content plays a crucial role in attaining the preferred self-healing capabilities. HPU/SNP1 and HPU/SNP1.5 showed rapid self-healing within $20 \mathrm{~s}$ under $M W$ radiation whereas HPU did not undergo self-healing behavior. This could be due to the fact that the energy required for polymeric chain activation to heal its surface is less as compared to the diffusion of the polymeric chains at the cracked surface [36]. Conversely, f-SNP has better $M W$ absorbing ability due to which it can transfer more amount of energy to the HPU matrix of the nanocomposites. This leads to enhanced Micro-Brownian movement of the soft segment of HPU nanocomposite and diffusion in the cracked places and thus healing it. It is also observed that the tensile strength of the nanocomposite remained same even after the healing experiment under

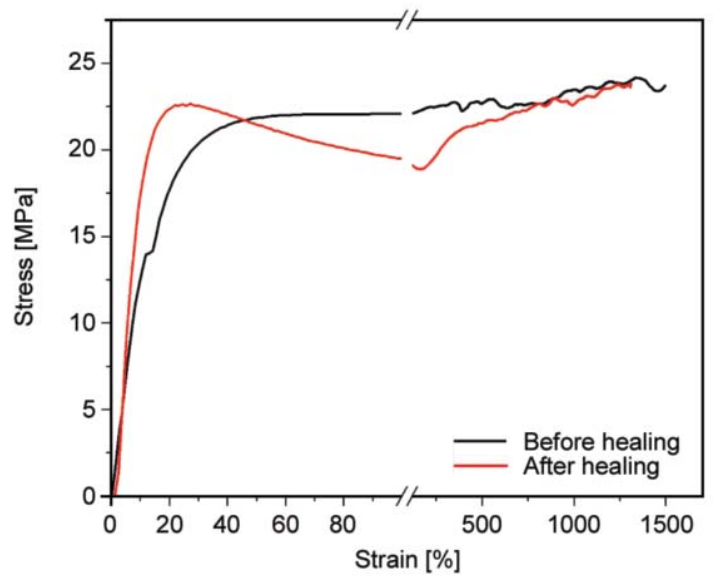

Figure 11. Representative stress-strain profiles of HPU/ SNP1, before and after healing.

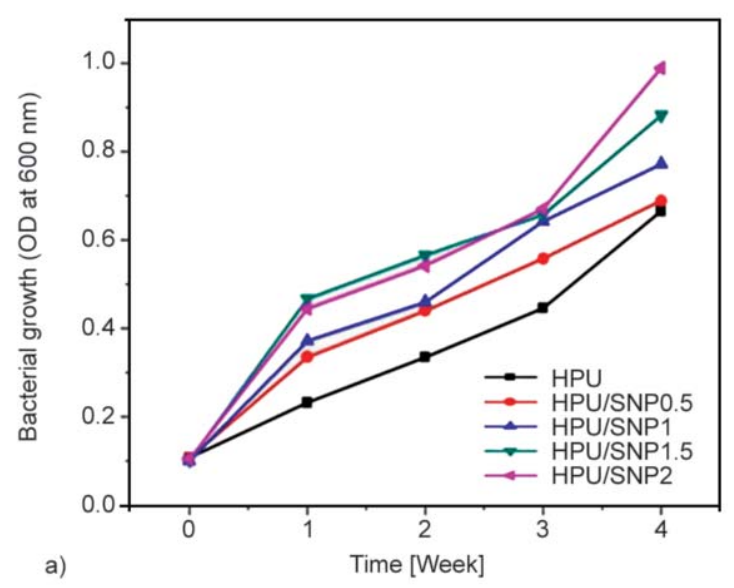

$M W$. Representative stress-strain curves before and after healing of HPU/SNP1 is shown in Figure 11.

\subsection{Biodegradation test}

From the biodegradation, it is confirmed that HPU and its nanocomposites are regularly degraded upon exposure to both $P$. aeruginosa and $B$. subtilis bacterial strains (Figure 12). The differences in the cell wall structures of Gram-positive bacteria $B$ subtilis and Gram-negative bacteria, P. aeruginosa, act as active barriers that control the degradation of the polymer substrate. It was observed that HPU films were considerably degraded after 4 weeks of inoculation by the respective bacterial strains, which signifies that the polymer acts as a catabolite (carbon source) to the bacteria. The degradation was rapid for all the nanocomposites in comparison to HPU. The incorporation of f-SNP enhances the rate of biodegradation as f-SNP is biocompatible and non-toxic [37, 38]. HPU/SNP2 shows the maximum optical density in both the cases, after a period of 4 weeks as it contains the highest amount of f-SNP which makes it more susceptible to bacterial growth, as apparent from the OD curves. The HPU films also showed a reduction in their weights after 4 weeks of exposure. This is mainly attributed to dissolution, bleaching, and degradation of starch by the bacterial attack. Thus, from the biodegradation study it can be concluded that HPU and its nanocomposites are biodegradable and the rate of biodegradation is dependent on f-SNP and starch content.

\section{Conclusions}

In conclusion, we established a smart bio-based hyperbranched polyurethane nanocomposite with functionalized silica nanoparticles through a simple, facile

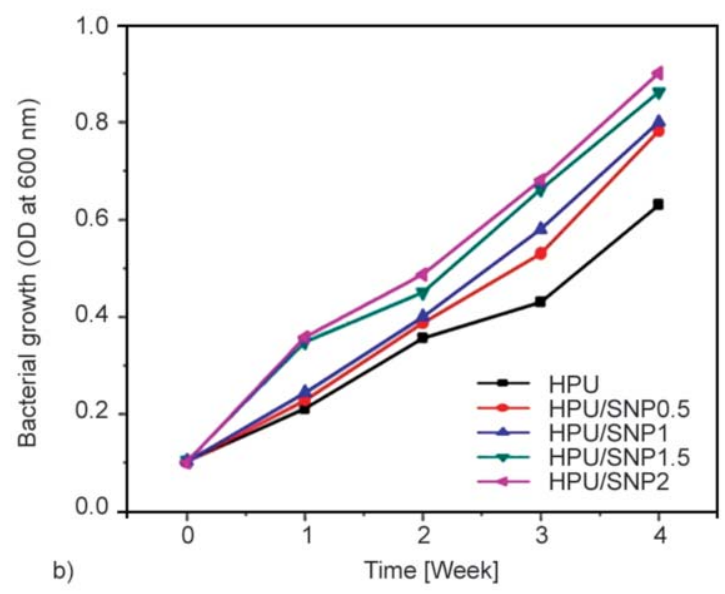

Figure 12. Biodegradation of HPU and its nanocomposites against (a) Bacillus subtilis and (b) Pseudomonas aeruginosa. 
one-pot process. The fabricated nanocomposite exhibited good thermal stability, mechanical properties, and hydrophobicity. The nanocomposites also demonstrated rapid self-healing ability under microwave exposure. Moreover biodegradability of the fabricated nanocomposite can solve solid waste management issues, and land and water pollution problems. Thus, the fabricated nanocomposite holds significant promise as a sustainable material for its prospective advanced industrial applications.

\section{Acknowledgements}

The authors would also like to thank Sophisticated Analytical Instrument Facility (SAIF), North-Eastern Hills University (NEHU) for helping with the TEM analysis and Sophisticated Analytical Instrumentation Centre (SAIC) Tezpur University for the different analyses used in this study.

\section{References}

[1] Montemor M. F.: Functional and smart coatings for corrosion protection: A review of recent advances. Surface and Coatings Technology, 258, 17-37 (2014). https://doi.org/10.1016/j.surfcoat.2014.06.031

[2] De las Heras Alarcón C., Pennadam S., Alexander C.: Stimuli responsive polymers for biomedical applications. Chemical Society Reviews, 34, 276-285 (2005). https://doi.org/10.1039/B406727D

[3] Li X-M., Reinhoudt D., Crego-Calama M.: What do we need for a superhydrophobic surface? A review on the recent progress in the preparation of superhydrophobic surfaces. Chemical Society Reviews, 36, 1350-1368 (2007).

https://doi.org/10.1039/b602486f

[4] Farhadi S., Farzaneh M., Kulinich S. A.: Anti-icing performance of superhydrophobic surfaces. Applied Surface Science, 257, 6264-6269 (2011). https://doi.org/10.1016/j.apsusc.2011.02.057

[5] Li Y., Chen S., Wu M., Sun J.: All spraying processes for the fabrication of robust, self-healing, superhydrophobic coatings. Advanced Materials, 26, 3344-3348 (2014). https://doi.org/10.1002/adma.201306136

[6] Duarah R., Karak N.: High performing smart hyperbranched polyurethane nanocomposites with efficient self-healing, self-cleaning and photocatalytic attributes. New Journal of Chemistry, 42, 2167-2179 (2018). https://doi.org/10.1039/C7NJ03889E

[7] Chen Y., Kushner A. M., Williams G. A., Guan Z.: Multiphase design of autonomic self-healing thermoplastic elastomers. Nature Chemistry, 4, 467-472 (2012). https://doi.org/10.1038/nchem.1314
[8] Huang L., Yi N., Wu Y., Zhang Y., Zhang Q., Huang Y., Ma Y., Chen Y.: Multichannel and repeatable self-healing of mechanical enhanced graphene-thermoplastic polyurethane composites. Advanced Materials, 25, 2224-2228 (2013). https://doi.org/10.1002/adma.201204768

[9] Puretskiy N., Stoychev G., Synytska A., Ionov L.: Surfaces with self-repairable ultrahydrophobicity based on self-organizing freely floating colloidal particles. Langmuir, 28, 3679-3682 (2012).

https://doi.org/10.1021/la204232g

[10] Puretskiy N., Stoychev G., Stamm M., Ionov L.: Switchable surfaces based on freely floating colloidal particles. ACS Applied Materials and Interfaces, 2, 2944 2948 (2010). https://doi.org/10.1021/am100634m

[11] Li Y., Li L., Sun J.: Bioinspired self-healing superhydrophobic coatings. Angewandte Chemie International Edition, 49, 6129-6133 (2010). https://doi.org/10.1002/anie.201001258

[12] Pan S., Kota A. K., Mabry J. M., Tuteja A.: Superomniphobic surfaces for effective chemical shielding. Journal of the American Chemical Society, 135, 578-581 (2012).

https://doi.org/10.1021/ja310517s

[13] Tropmann A., Tanguy L., Koltay P., Zengerle R., Riegger L.: Completely superhydrophobic PDMS surfaces for microfluidics. Langmuir, 28, 8292-8295 (2012). https://doi.org/10.1021/la301283m

[14] Liu X., Xu Y., Chen Z., Ben K., Guan Z.: Robust and antireflective superhydrophobic surfaces prepared by CVD of cured polydimethylsiloxane with candle soot as a template. RSC Advances, 5, 1315-1318 (2015). https://doi.org/10.1039/C4RA12850H

[15] Chakradhar R. P. S., Kumar V. D., Rao J. L., Basu B. J.: Fabrication of superhydrophobic surfaces based on ZnO-PDMS nanocomposite coatings and study of its wetting behaviour. Applied Surface Science, 257, 85698575 (2011). https://doi.org/10.1016/j.apsusc.2011.05.016

[16] Seyfi J., Jafari S. H., Khonakdar H. A., Sadeghi G. M. M., Zohuri G., Hejazi I., Simon F.: Fabrication of robust and thermally stable superhydrophobic nanocomposite coatings based on thermoplastic polyurethane and silica nanoparticles. Applied Surface Science, 347, 224-230 (2015).

https://doi.org/10.1016/j.apsusc.2015.04.112

[17] Chen Y., Zhou S., Yang H., Wu L.: Structure and properties of polyurethane/nanosilica composites. Journal of Applied Polymer Science, 95, 1032-1039 (2005). https://doi.org/10.1002/app.21180

[18] Duarah R., Singh Y. P., Mandal B. B., Karak N.: Sustainable starch modified polyol based tough, biocompatible, hyperbranched polyurethane with a shape memory attribute. New Journal of Chemistry, 40, 5152-5163 (2016).

https://doi.org/10.1039/C5NJ03294F 
[19] Duarah R., Singh Y. P., Gupta P., Mandal B. B., Karak N.: High performance bio-based hyperbranched polyurethane/carbon dot-silver nanocomposite: A rapid selfexpandable stent. Biofabrication, 8, 045013/1045013/21 (2016).

https://doi.org/10.1088/1758-5090/8/4/045013

[20] Gogoi S., Karak N.: Biobased biodegradable waterborne hyperbranched polyurethane as an ecofriendly sustainable material. ACS Sustainable Chemistry and Engineering, 2, 2730-2738 (2014).

https://doi.org/10.1021/sc5006022

[21] Loganathan S., Sankaran S.: Surface chemical studies on silicon carbide suspensions in the presence of poly (ethylene glycol) and chitosan. Colloid and Surface Science, 2, 6-20 (2017).

https://doi.org/10.11648/j.css.20170201.12

[22] Rao G. R.: Infrared reflection absorption study of water interaction with $\mathrm{H}$-terminated $\mathrm{Si}(100)$ surfaces. Bulletin of Materials Science, 27, 497-500 (2004).

https://doi.org/10.1007/BF02707275

[23] Nallathambi G., Ramachandran T., Rajendran V., Palanivelu R.: Effect of silica nanoparticles and BTCA on physical properties of cotton fabrics. Materials Research, 14, 552-559 (2011).

https://doi.org/10.1590/S1516-14392011005000086

[24] Barron M. K., Young T. J., Johnston K. P., Williams R. O.: Investigation of processing parameters of spray freezing into liquid to prepare polyethylene glycol polymeric particles for drug delivery. AAPS PharmSciTech, 4, 1-13 (2003) https://doi.org/10.1208/pt040212

[25] Yu D-G., Zhu L-M., Branford-White C. J., Yang J-H., Wang X., Li Y., Qian W.: Solid dispersions in the form of electrospun core-sheath nanofibers. International Journal of Nanomedicine, 6, 3271-3280 (2011). https://doi.org/10.2147/IJN.S27468

[26] Kwon N. K., Kim H., Han I. K., Shin T. J., Lee H-W., Park J., Kim S. Y.: Enhanced mechanical properties of polymer nanocomposites using dopamine-modified polymers at nanoparticle surfaces in very low molecular weight polymers. ACS Macro Letters, 7, 962-967 (2018).

https://doi.org/10.1021/acsmacrolett.8b00475

[27] Thakur S., Karak N.: Tuning of sunlight-induced selfcleaning and self-healing attributes of an elastomeric nanocomposite by judicious compositional variation of the $\mathrm{TiO}_{2}$-reduced graphene oxide nanohybrid. Journal of Materials Chemistry A, 3, 12334-12342 (2015). https://doi.org/10.1039/C5TA02162F

[28] Ou X., Lin Z., Li J.: Surface microstructure engenders unusual hydrophobicity in phyllosilicates. Chemical Communications, 54, 5418-5421 (2018).

https://doi.org/10.1039/C8CC02102C
[29] Cai Q., Huang J., Weng R., Liu S.: Preparation and surface properties of silicon-containing waterborne polyurethane functionalized with fluorine-containing acrylate and micro-nano silica. Journal of Wuhan University of Technology-Materials Science Edition, 33, 233-241 (2018).

https://doi.org/10.1007/s11595-018-1811-2

[30] Bhushan B., Jung Y. C., Koch K.: Micro-, nano- and hierarchical structures for superhydrophobicity, self-cleaning and low adhesion. Philosophical Transactions of the Royal Society A: Mathematical, Physical and Engineering Sciences, 367, 1631-1672 (2009).

https://doi.org/10.1098/rsta.2009.0014

[31] Ghosh T., Karak N.: Tough interpenetrating polymer network of silicone containing polyurethane and polystyrene with self-healing, shape memory and self-cleaning attributes. RSC Advances, 8, 17044-17055 (2018). https://doi.org/10.1039/C8RA01766B

[32] Cho J. W., Lee S-H.: Influence of silica on shape memory effect and mechanical properties of polyurethanesilica hybrids. European Polymer Journal, 40, 13431348 (2004). https://doi.org/10.1016/j.eurpolymj.2004.01.041

[33] Wang J., van Tittelboom K., de Belie N., Verstraete W.: Use of silica gel or polyurethane immobilized bacteria for self-healing concrete. Construction and Building Materials, 26, 532-540 (2012).

https://doi.org/10.1016/j.conbuildmat.2011.06.054

[34] Liu Q., Wang X., Yu B., Zhou F., Xue Q.: Self-healing surface hydrophobicity by consecutive release of hydrophobic molecules from mesoporous silica. Langmuir, 28, 5845-5849 (2012).

https://doi.org/10.1021/la300187q

[35] Thakur S., Barua S., Karak N.: Self-healable castor oil based tough smart hyperbranched polyurethane nanocomposite with antimicrobial attributes. RSC Advances, 5, 2167-2176 (2015). https://doi.org/10.1039/C4RA11730A

[36] White S. R., Sottos N. R., Geubelle P. H., Moore J. S., Kessler M., Sriram S. R., Brown E. N., Viswanathan S.: Autonomic healing of polymer composites. Nature, 409, 794-797 (2001). https://doi.org/10.1038/35057232

[37] Bari S. S., Chatterjee A., Mishra S.: Biodegradable polymer nanocomposites: An overview. Polymer Reviews, 56, 287-328 (2016).

https://doi.org/10.1080/15583724.2015.1118123

[38] Patwary F., Mittal V.: Degradable polyethylene nanocomposites with silica, silicate and thermally reduced graphene using oxo-degradable pro-oxidant. Heliyon, 1, e00050/1-e00050/21 (2015). https://doi.org/10.1016/j.heliyon.2015.e00050 